\title{
Imaginative Resistance and Modal Knowledge
}

\author{
Daniel Nolan
}

Final version to appear in Res Philosophica.

\begin{abstract}
Readers of fictions sometimes resist taking certain kinds of claims to be true according to those fictions, even when they appear explicitly or follow from applying ordinary principles of interpretation. This "imaginative resistance" is often taken to be significant for a range of philosophical projects outside aesthetics, including giving us evidence about what is possible and what is impossible, as well as the limits of conceivability, or readers' normative commitments. I will argue that this phenomenon cannot do the theoretical work that has been asked of it. Resistance to taking things to be fictional is often best explained by unfamiliarity with kinds of fictions than any representational, normative, or cognitive limits. With training and experience, any understandable proposition can be made fictional and be taken to be fictional by readers. This requires a new understanding both of imaginative resistance, and what it might be able to tell us about topics like conceivability or the bounds of possibility.
\end{abstract}

An undeniable feature of our imaginative life is that we do not always successfully imagine things we try to imagine, and an equally familiar feature of our imagination is that some attempts to imagine are more challenging than others. Related to these phenomena is a phenomenon involving the uptake of fiction. When consuming fiction we are often willing to take a text's sayso as definitive about what is going on in a story. Sometimes, however, when something is presented as being true according to a story, some people balk at treating it as in fact being true according to the relevant story. Again, this phenomenon comes in degrees: as well as the phenomenon of just not taking something to be true according to a fiction, sometimes we will in the end accept that something is true according to a story but find it hard to make sense of; or be reluctant to incorporate it in our understanding of the story; and so on.

It would have been ideal if we had different names for this resistance in imagination and this resistance in fiction, but the literature blurs them together. ${ }^{1}$ One reason for blurring them together is the idea that we might explain the phenomenon involving fiction by appeal to the phenomenon

\footnotetext{
${ }^{1}$ There are some proposals to give these phenomena different labels: for example Weatherson 2004 distinguishes the "alethic puzzle" and the "imaginative puzzle" which are why the relevant propositions are resisted as being true according to a fiction or imagined, respectively; while Stear 2015 distinguishes "imaginative failure" and "fictionality failure", closely related to the two relevant kinds of resistance. Neither terminological proposal has yet caught on generally.
} 
involving imagination: a simple story would be that taking $p$ to be true according to a fiction amounts to imagining that $p$ when engaging with the fiction, and so when we cannot imagine $p$, we cannot take it to be true according to a fiction either. Likewise, when we find it difficult to imagine $p$ we would find it difficult to take to be true according to a fiction, and so on. That story of the connection is no doubt overly crude; even someone who wants to treat engagement with fiction as involving imagination is unlikely to demand a connection that is that tight. There are very many ways to "engage with fiction", after all, and some do not seem to require much imagination. (Remembering the right answer in a multiple-choice test on Hamlet may not require much imaginative engagement with Shakespeare's story, for example.)

The aspect of this discussion I want to focus on in this paper is the question about resistance to taking things to be true according to fictions. Roughly speaking, the phenomenon I am interested in is the tendency for consumers of fiction ("readers", from here on), to not take things to be true according to fictions, or to be "part of the story", even when those propositions are presented explicitly as part of the story, or would seem to be part of the story given straightforward ways we supply the implicit content of fictions. Tamar Gendler (2000 p 61) labels it the "that's what you think!" phenomenon: while we are often willing to take a storyteller's say-so for what is going on in a story, there are various kinds of cases where readers do not do this. This characterisation is fairly rough at this stage, and it is hard to be too precise without settling for particular accounts of when something is explicit in a story, or when something would be delivered by straightforward mechanisms of generating implicit content. Despite the tangled history of the expression, I will use "imaginative resistance" from now on to talk about the phenomenon related to interpretation of fiction, rather than questions explicitly about what people imagine in response to fictions.

Apart from the intrinsic interest of questions about how people respond to fictions, and what it takes for a claim to be true according to a fiction, there are several reasons imaginative resistance is philosophically interesting. One important reason to be interested is that, if the views of some theorists are right, the limits of what we can take to be fictional might tell us about the limits of possibility. According to theorists like Lewis 1986 and Stalnaker 1984 and Jackson 2011, the limits of what can be represented just are the limits of possibility, insofar as propositional 
representation is always a matter of drawing distinctions among possibilities. According to those theorists, there is only one impossible proposition (corresponding to the empty set of possible worlds), and this proposition entails every other proposition. That strongly suggests that we cannot take discriminating attitudes to one impossible content rather than another, including taking some but not all impossibilities to be true according to a fiction. On the other hand, when we can take a proposition $p$ to be true according to a fiction without taking all impossibilities to be true according to that fiction, that strongly suggests, given the picture of content offered by these theorists, that we are dealing with a possibility. ${ }^{2}$ On the face of it, at least, lack of imaginative resistance should be a mark of the possible if this picture of content is right.

The connection between taking something to be true in fiction, on the one hand, and imaginable or conceivable, on the other, is a contested one, not least because debates about "imaginability" or "conceivability" are often between parties with different standards for applying those expressions. Even once we set aside senses of "imagination" which primarily involve sensory imagination (and the associated phenomenology of something-like-sensation that goes with vivid imagination, in this sense), disagreement will remain about how much interpretation of fiction requires imagining, or even conceiving. Presumably our engagement with fiction has something useful to tell us about imagination and conceiving. In turn, information about the limits of conceivability may indirectly tell us about the limits of what is possible, since a popular thought is that conceivability is a good epistemic guide to possibility, and in addition something's being inconceivable is a good evidence that it is impossible. I will return to a discussion of connections with conceiving, below.

A different way patterns of imaginative resistance might be informative in uncovering the normative and evaluative commitments of readers. Weatherson 2004, building on remarks in Yablo 2002, points out that we may be especially reluctant to take an author's say-so about aesthetic, moral or character evaluations when we are given descriptions in non-evaluative terms that seem to conflict with those evaluations. Yablo 2002 p 485 gives a memorable example of a vignette in which (allegedly) the exhilarating and profound beauty of a monster truck rally is

\footnotetext{
2 There are many other reasons to think that fictions can represent some impossibilities without thereby representing them all: for discussion see e.g. Priest 1997 and Nolan 2015.
} 
spoiled by a sunset. He points out, reasonably, that we might have no difficulty taking it to be part of the story that a monster truck rally is occurring at sunset, but we may have much more difficulty taking the author's word for it that the scene as described is beautiful, or marred by a sunset. Some have hoped that we can explain our pattern of imaginative resistance by appeal to our normative commitments (see Gendler 2000 for the moral case); but were this connection robust, we could also use an examination of the pattern of imaginative resistance to tell us interesting things about those normative commitments as well. Other commitments besides normative and evaluative ones may also manifest themselves in patterns of imaginative resistance if Yablo 2002 and Weatherson 2004 are right: I will also discuss these below.

Despite the hopes many theorists have had for using imaginative resistance to tell us about other topics of interest, I think the correct explanation of much imaginative resistance will reveal that it is not suitable to do the ambitious theoretical work that has been asked of it. In my view, much more can be taken to be true according to fictions, with little or no resistance, than many have thought. Indeed, for virtually all propositions $p$, standard readers can be brought to take $p$ to be true of a fiction (or at least a reading of a fiction), without resistance. If this is right, then looking for a special range of propositions readers will resist is a mistake. Theories that suppose that there is such a special range and go on to argue that they have special features (impossibility, inconceivability, clash with our normative commitments, etc.) will be mistaken as well. Looking to patterns of imaginative resistance will be of very limited use, at best, in the epistemology of modality. Our flexibility with fiction also suggests many impossible propositions are conceivable: and if this is so, accounts of modal epistemology that lean on conceivability will also be in trouble.

\section{Refining the Target}

The early literature on imaginative resistance focused on moral content of stories, especially stories where actions are described as right or good or just which we would consider anything but. One stock example is a very simple one from Walton 1994 p 37, the case of Giselda: "In killing her baby, Giselda did the right thing; after all, it was a girl". In a story without much other context, this strikes many (or at least, many of those who have written about it) as not being a 
story fragment where Giselda did the right thing in killing her baby. Maybe it is a story where Giselda thought she was doing the right thing, or the implied author thought she was doing the right thing, or... but not one where we take the story's say-so that Giselda did the right thing.

Others soon pointed out that something similar seems to happen with other kinds of stories. We can have imaginative resistance when something that sounds hideous is described as elegant or beautiful. (Yablo 2002 p 285) Authors sometimes present their own poetry or song lyrics and describe them as haunting or beautiful; and while we may be willing to think that the poems or songs strike the characters as having great aesthetic value, we may be less willing to take clumsy doggerel to be beautiful, even in the story, on an author's say-so. Imaginative resistance does not seem to be confined to the normative or evaluative either. We can have imaginative resistance when authors violate some conceptual connections or other fundamental connections we take to hold between matters described in different vocabularies: we are not willing to accept that a shape that is jagged and matches our stereotype of being a maple leaf could nevertheless be oval in a story (Yablo 2002 p 485), or that a train could have carriages added to them without those carriages becoming part of the resulting train (Weatherson $2004 \mathrm{p} \mathrm{5).} \mathrm{The} \mathrm{boundaries} \mathrm{of} \mathrm{this}$ phenomenon of conceptual-constraint-violation producing resistance are disputed, but at least some supposed cases of this seem to baffle many readers who encounter them.

Once we are talking about uptake of fictions, it is worth taking note of the fact that different readers interpret fictions differently, and even a single reader can take a given fiction in more than one way. When we are asking about what readers take to be true according to fictions, there is often no single answer, particularly when fictions are puzzling or challenging. I think it is worthwhile here to talk about the readings that are available to a reader of a given fiction. As is well known, readers can deliberately take up stances towards texts to extract stories from those texts that may have been very different from the one(s) the author intended, or indeed the ones that would normally be drawn from the text. These readings include "resistant readings" where readers set aside the evaluative judgements the author seems to intend and substitute their own evaluations or political commitments to characterise what happens. (See e.g. Fetterley 1978.) But they also involve other playful readings, of all sorts: infusing relatively staid narratives with erotic subtexts, reading The Lord of the Rings as an extended Dungeons and Dragons session, 
and so on. When asking whether there is imaginative resistance to a given proposition relative to a fiction, I think it is best to enquire about whether a reader can find that proposition to be true according to any of the readings of the text a reader is willing to countenance. Distinguishing readings helps us do justice to two important facts about approaching stories written from viewpoints with which we disagree: both that we can read resistantly, imposing our own values and assumptions on the events described, and that we can read exploratively or sympathetically, trying to see the story from the author's own point of view, or uncovering the story the author herself was trying to tell. Relativising what is true according to a fiction to readings of that fiction gives us a way to do justice to both of those ways we can engage with stories, and to the many other appearances stories have to readers besides.

With that background in place, I am in a position to offer a definition of imaginative resistance, in the sense involving taking things to be fictional rather than the sense directly focused on imagination.

Imaginative Resistance: In cases where a proposition might, by normal standards, seem to be true according to a fiction, there is imaginative resistance when readers nevertheless are reluctant, unwilling, or unable to take that proposition to be true according to the fiction, on any reading available to them.

I will not try to give an account of when a proposition would be true according to a fiction (or be true according to a reading of a fiction) by normal standards. I want to include cases where there is an explicit claim in a fiction ("and it was the right thing to do..."), but also a range of cases where a proposition is presented implicitly, since so much of the content of fictions is presented in this way. It might be through an approving tone of an implied narrator, what is said by characters represented as well informed or as experts, and so on. A full story of what it takes, even typically, for a fiction to represent a proposition may need to await an adequate and general theory of fiction itself. But hopefully relying on our sense of how fictions normally present propositions will do for present purposes. 
Once we define imaginative resistance this way, there will be a number of boring reasons we might find imaginative resistance in a particular case. A fiction may rely on unfamiliar concepts, or too much unknown background information. It may contain too much information or be too unengaging to slog through. (Many readers who attempt to engage with Tolstoy's War and Peace do not engage with the second half of the work, but only because they do not get that far.) Consumers of fiction may also fail to take propositions to be true according to a fiction because the fiction is too distasteful or unpleasant to engage with, or to be worth paying enough attention to to draw out those details. Another reason a reader may fail to take a proposition to be true according to a given fiction because the proposition is too hidden or indirectly presented: an author who scatters clues through too many red herrings, or who is too allusive or layered, may produce a work that has content that is not picked up by most of its readers.

Call imaginative resistance that comes from these sources mundane imaginative resistance. The psychological study of engagement with fiction cannot afford to neglect these phenomena. (And indeed someone with an eye to e.g. producing more compelling advertisements might be primarily interested in removing those sources of resistance.) But the fact that people might be reluctant, unwilling or unable to take certain things to be true according to a fiction because they are bored, or nauseated, or confused by the intricacy of the text is unlikely to be significant for our theoretical purposes. Even when we set mundane imaginative resistance aside there are still many cases calling for explanation. Let us now turn to some attempts to understand what is going on when readers are unwilling or unable to go along with how stories appear to characterise fictional goings-on, in cases analogous to the Giselda or shape cases discussed above.

\section{Some Approaches to Imaginative Resistance}

The two main camps in the literature diagnosing the cause of (philosophically interesting) imaginative resistance are, in the taxonomy and terminology of Gendler and Liao 2016, the "cantian" and "wontian" theories of imaginative resistance. According to "cantians", readers lack the ability to take certain kinds of propositions to be true according to fictions, or alternatively they lack the ability to imagine certain propositions to be the case, and this unimaginability leads 
to their being unable to take those propositions to be true according to fictions. Typically cantians relativise which propositions these are to readers, at least to some extent: tying it to readers' beliefs, inferential dispositions, or other capacities.

Brian Weatherson (Weatherson 2004) proposes one example of a "cantian" theory. According to Weatherson, interesting cases of imaginative resistance arise because of inferential dispositions readers have from information about "lower level" facts to conclusions about "higher level" facts that follow from them. Examples include moral conclusions from descriptive premises (once you tell me a mother killed her child just for some peace and quiet, you've told me enough to work out whether what she did was wrong), other evaluative matters (including what is beautiful, funny, etc.) and also connections between sets of descriptive claims: from the claim that something is five-pointed and jagged, it follows it is not oval-shaped; and from the fact that a train engine is pulling train carriages, it follows there is a train that has some carriages as parts. And so on. So the kind of resistance Weatherson predicts must occur will be where there are apparent violations of a constraint Weatherson calls "Virtue":

\section{Virtue}

If $p$ is the kind of claim that, if true, must be true in virtue of lower-level facts, and if the story is about those lower-level facts, then it must be true in the story that there is some true proposition $r$ which is about those lower-level facts such that $p$ is true in virtue of $r$. (Weatherson $2004 \mathrm{p} 18$ )

Or rather, fictional interpretations must reflect the reader's opinions about what it is in virtue of which $p$ holds. I take it the right way to read his principle is not just that the story says that $p$ is true in virtue of $r$, but that $r$ is in fact a proposition that $p$ could, or would, be true in virtue of. Weatherson says of Virtue only that it is a "strong default principle of fictional interpretation" (18), which does leave room for holding that there are cases where it is overridden. Too many such cases, and Weatherson will not only arguably not count as a cantian (if we can too easily take things to be true according to fictions that his principle apparently rules out), but also may lose a lot of the explanatory force of his hypothesis. Note that the kinds of propositions Virtue predicts will be resisted will typically be complex ones: not just that Giselda did the right thing in killing her child, but that Giselda did the right thing in killing her child just because it was a 
girl. What will be resisted are (most typically) propositions that express combinations of higherlevel truths with lower-level truths. Specifically, combinations of higher order truths with specifications of lower level truths that rule out all of the sets of lower-level truths that those higher-level truths could obtain in virtue of. (For instance, what is resisted is not just that a thing is oval shaped, but that it is overall oval shaped even though aspects of its shape include being five-pointed and jagged).

Gendler 2000, on the other hand, presents an influential "wontian" theory of imaginative resistance according to which it is not the case that readers cannot make sense of the scenarios that fictions present; it is rather that when these scenarios presuppose commitments to normative claims the reader rejects, readers refuse to operate the capacity which they have to treat the author's say-so as definitive. Gendler argues that we can make sense even of stories that represent impossibilities, such as a story where God changes the principles of mathematics, so imaginative resistance is not due to some general inability to include logical or metaphysical impossibilities in our readings of fictions. Instead, according to Gendler,

$[\mathrm{C}]$ ases that evoke genuine imaginative resistance will be cases where the reader feels that she is being asked to export a way of looking at the actual world which she does not wish to add to her conceptual repertoire. (Gendler 2000 p 77)

In the moral cases, we are able to understand fictional moral truths that vary widely from the judgments we might non-fictionally make in such circumstances; but Gendler argues that we often find such judgments distasteful or fear them as potentially corrupting. Gendler's theory appears to predict that when a fiction embodies a system of evaluations (particularly moral ones, but we could generalise the proposal to many other evaluative topics), those who do not want to become familiar or conversant with that way of approaching matters will imaginatively resist. ${ }^{3}$

\footnotetext{
${ }^{3}$ Gendler significantly modifies her position in Gendler 2006, expanding the account to cover other evaluations, and generalising it to cover apparently descriptive cases of the sort Yablo 2002 and Weatherson 2004 discuss. In that paper, Gendler is more explicit that a very wide range of propositions can be true according to stories and imaginable given enough "authorial ingenuity". "With enough ingenuity, these resources can, I suspect, be used to make any local bit of content not only true in a story, but also straightforwardly imaginable". (p 158 ftnt 24) While I focus more on resources readers might have than authors might deploy, I suspect by 2006 Gendler may not disagree with the central thrust of this paper.
} 
Incidentally, "adding a way of looking at the world to our conceptual repertoire" is doing a lot of work here, and it is hard to see how to interpret it in a way that does justice to the cases. I understand a variety of moral and evaluative views that I do not share, and know how to go on with them at least well enough to predict and explain those people who in fact hold those views. Since those "ways of looking at the actual world" already appear to be part of my conceptual repertoire, it is hard to see how I could experience imaginative resistance when exposed to fiction with racist or misogynist messaging, for example. (After all, racist and misogynist ways of "looking at the world" are all too familiar.) But I do sometimes resist readings where protagonists are supposed to be behaving correctly when they engage in racist or misogynistic behaviour. So if the standard for "adding to a conceptual repertoire" is just understanding the way of seeing and knowing how to go on with it, Gendler's theory appears to under-predict imaginative resistance, since I sometimes resist when I already possess the relevant conceptual capacities (and so do many other readers). On the other hand, if the relevant "export" is a matter of coming to endorse the relevant evaluative claims, the theory makes imaginative resistance puzzling: if I do not feel the need to fail to understand people when they ask me to change my evaluative views in other ways, why would I have to resist readings of fictions when I know the author intends the fiction as part of a case for changing my evaluations? At any rate, wontian theories in general need not trace the reluctance to engage in readings to exactly the sources Gendler identifies - the common thread is that readers will be capable of reading certain propositions as true in fictions, but will not treat them as true in such fictions even when apparently invited to by the text. Which propositions these are depends on what commitments a reader brings to the fiction.

Wontian theories work most straightforwardly when dealing with normative or evaluative propositions that are supposed to be true according to fictions. But we could extend such proposals to the other sorts of imaginative resistance discussed in the literature. Readers have other inferential principles they use besides transitions from descriptions to evaluations, and they might resist compromising them even in fictional contexts. This might apply to the connections Weatherson describes, above, such as between information about the distribution of matter and the application of shape predicates, matters of fact and "metaphysical" consequences of them like questions of what is part of what, and so on. We could even go beyond existing wontian theories 
and conjecture that this sort of reluctance to suspend inferences can be what is going on in many cases of imaginative resistance that go along with complaints about "unrealistic" fictions. Some people with extensive scientific backgrounds cannot enjoy "soft" science fiction because of the gross contrary-to-fact features of the physics or other scientific facts about the fictional worlds described. Most consumers can handle stories of etheric winds or telekinetic energy, but some do not or will not; and a selective "wontian" theory looks more plausible for these cases than the corresponding "cantian" theory according to which people who do not suspend their assumptions that actual-world physics are in play lack some capacity the rest of us have.

In opposition to both the "cantian" and "wontian" camps, I think the explanation of non-mundane imaginative resistance is not to be found in either a deep-seated inability in readers to treat certain propositions as parts of stories, nor in a general disposition to refuse to engage with stories embodying attitudes the reader finds distasteful or otherwise rejects. Instead, nonmundane imaginative resistance can be removed through readers gaining more education and experience. Readers familiar with a wide enough range of genres of fiction, and aware enough that authors may not share the readers' background assumptions, can be brought to interpret fictions so that various surprising propositions are true according to those fictions after all. In this paper I will defend a strong thesis: that for any understandable proposition $p$, readers with wide enough horizons can take $p$ to be true according to suitably constructed and presented fictions. The distinction between claims that evoke imaginative resistance and those that do not has less to do with the content of any particular claim, and more to do with how familiar readers are with different kinds of demands a variety of fiction might make on them. I should stress that readers can gain this familiarity without necessarily corrupting their moral or other evaluative commitments: so in my view "wontian" theories attribute too much interpretational inflexibility to readers, even holding fixed firm commitments by those readers to moral, aesthetic, or other values. Consider an analogy: travel broadens the mind, but it does not follow that travel must be corrupting.

This diagnosis has some points of similarity with the position advanced by Todd 2009. Todd claims to "dissolve" the puzzle of imaginative resistance, though he seems to have in mind a somewhat different puzzle from the ones I have been considering, involving an alleged 
asymmetry between imaginative resistance towards certain moral propositions and certain nonmoral ones. Todd claims that the presuppositions of this puzzle dissolve once we notice 1) the role that the commitments of readers play to what they are going to resist and what they will allow is true in a fiction and 2) that we should be "interpretivist" about fictional truth rather than "realist" about fictional truth (Todd 2009 p 188-9). I am not entirely sure what Todd intends by the latter, though I think it involves at least in part rejecting the idea that there is a single onceand-for-all answer to the question of what is true according to a given fiction, and that there is a single set of principles that we should follow in determining what we take to be going on in a story.

I do not think the interesting puzzles raised by the literature on imaginative resistance disappear if we allow that reader's dispositions can vary in the way Todd suggests and that truth in fiction is somehow interpretative. In particular, we can still raise the question of whether there are understandable propositions $p$ such that readers will resist taking them to be true according to any fiction (even if this taking is properly "interpretive" rather than "realist", however exactly Todd is thinking of this contrast.) However, I do want to agree with Todd that there will be a wide variation of readers' responses to a given text, and that recognition that a variety of readings are possible should help us realise that there might be less imaginative resistance to particular propositions than we would expect if each reader is capable of no more than one reading of any particular text, let alone if we were to think (as not even realists about fictional truth would) that every text bears only one reading. More importantly, I want to agree with Todd (2009) p 193's suggestion that when we come across propositions that evoke imaginative resistance, we can often (always?) find another fiction that does not evoke such resistance, by adding context or altering readers' expectations.

However, the position in the literature that comes closest to the one I want to advance is that advanced in Liao, Strohminger and Sripada 2004. They suggest that contextual cues, including cues from genre expectations, can reduce imaginative resistance. Their focus is on moral cases, 
but I think the sort of position they have provided some experimental evidence for can be greatly expanded and generalised. ${ }^{4}$

\section{Liao, Strohminger and Sripada}

Research on imaginative resistance carried out by Shen-yi Liao, Nina Strohminger and Chandra Sripada (2004) points to one way imaginative resistance can be overcome, for a range of moral propositions that are supposed to provoke imaginative resistance in ordinary readers, at least by writers in the traditional "cantian" and "wontian" literatures. In one experiment, readers are presented with variations on stories where a mother gives up her newborn for human sacrifice. In one version of the story, it is presented as being in response to the demand of a contemporary cult leader, "Wayne Howell", whose name suggests he is American or at least Western. In the other version of the story, the demand for sacrifice comes from a High Priest Cihuacoatl, in what they were told was an Aztec creation myth. In this case neither story explicitly suggested a moral evaluation of what was done. Test subjects were much more likely to think the mother did the right think in giving up her baby for sacrifice in the latter story. (Test subjects thought that in both cases the mother believed she was doing the right thing, allowing us to be confident that the subjects did not confuse the question of what the right thing was in the story with e.g. what the characters in the story believed was the right thing.)

The lesson that Liao, Strohminger and Sripada draw here is that the influence of "genre" or "context" can make a difference to how much resistance there is to "morally deviant" propositions suggested by a fiction (353). That is, they think that moral propositions readers might ordinarily think are false can be taken to be part of a story by such readers, and one way to prompt them to do so is to make clear that the story is, or seems to be, from another time and place and produced by authors with different assumptions from the readers' own. Another experiment they report (348-350), asking participants for judgements about a story of Hippolytus

\footnotetext{
4 Another position in the literature similarly generous about what can be true according to fictions is that of van Inwagen 1993. There van Inwagen holds that "the author of a fantasy has power to confer 'truth in the story' on known conceptual falsehoods. I could, for example, write a fantasy in which there were two mountains touched at their bases but did not surround a valley". van Inwagen seems to suggest that even known conceptual falsehoods can, fairly straightforwardly, be made true according to stories, and presumably without facing imaginative resistance from competent readers.
} 
and Persephone, in which Hippolytus tricks Persephone into consuming a leaf that binds her to him for eternity, and in which Zeus pronounces the consequences just. Participants familiar with Greek myth were more likely to hold that Hippolytus's actions were the just thing to do in the story, even if they personally disapproved of the sort of thing he did.

There are reasons to be cautious of Liao et. al.'s conclusions, apart from broad methodological concerns about e.g. their relatively small sample sizes, and the fact that the results only seem to show that imaginative resistance is diminished rather than eliminated in the cases they test. Especially in the human sacrifice cases, we are not even told explicitly in the vignettes that the sacrifice is right, nor even that any god demands it: in the contemporary cult leader vignette, we might reasonably wonder whether anyone besides the cult leader demands it. It is not clear to me that in the vignette Liao et. al. offer about the cult leader, even an implied author or implied narrator would want us to take the sacrifice to be morally right, making it in some ways an unsuitable contrast to the "myth" where it seems more plausible that we are being asked, at least, to think the sacrifice is right. And we should be cautious in assimilating people's responses to (apparent) fiction to their responses to (apparent) myth: even though the "Aztec creation myth" Liao et. al. used was in fact a story they made up and not a genuine myth, if readers thought it was genuinely a myth they might use different techniques for telling what is true according to it. (We appear to use different techniques when interpreting fiction and interpreting various forms of non-fiction, after all; we are willing to take an author's say-so about what happened in a story, but less likely to take a historian's say-so about what happened in a contentious historical reconstruction.)

Whatever concerns we might have about the particular experiments Liao et. al. report, however, I think their results make a few things plausible. They make it plausible that cues from "context", broadly speaking, can modify how willing readers are to take things to be true according to stories, including when it comes to evaluative matters. These cues from context include assumptions readers can make about the origin of the fictions to be evaluated and the genre expectations for the kind of story they are dealing with. And their case of Hippolytus and Persephone gives us some preliminary evidence that wider familiarity with stories from other cultural backgrounds can also break down imaginative resistance. In my view, the phenomenon 
Liao et. al. have demonstrated is the tip of the iceberg. Readers can use cues about genre and the familiarity of the author's world-view to make better sense of stories as they are presented, and this phenomenon is already widespread across a range of actual readers' reactions. Furthermore, we have reason to think that imaginative resistance can be overcome in cases well beyond those where authors seem to be from cultural backgrounds that incorporate different moral assumptions: there are many different ways to construct fictions and train readers to incorporate propositions in readings without resistance.

\section{Expanded Horizons and Reduced Imaginative Resistance}

Liao et al. point out one way to lower imaginative resistance levels: once we remember other people do not think as we do, we are more open to understanding a story they tell as incorporating their presuppositions rather than our own. I believe this phenomenon is quite widespread: a common instance of it is when we read stories from cultures very different from our own. One of the most widely known stories in the history of Western culture is Homer's The Odyssey, and thousands of experts, not to mention tens or hundreds of thousands of nonspecialist readers, are familiar with many of the main events of the story. (It may be that The Odyssey was originally produced as non-fiction, but it is safe to say that most readers of it in the twentieth century consumed it as something closer to fiction, perhaps with factual elements buried within it.) Odysseus, the protagonist, is not presented as an entirely stereotypical hero, unlike Hector in The Iliad. But Odysseus is nevertheless presented sympathetically and as a hero for the most part.

When Odysseus returns home after many years at sea, he finds his household being bullied and menaced by a pack of suitors trying to convince his wife Penelope to marry one of them. We would think Odysseus has a right to be upset: and given the suitors' behaviour, we may even be willing agree that Odysseus, as protector of his household and the local legal authority in virtue of being king of Ithaca, could legitimately punish the suitors. So far, so good. Odysseus's response to this pack of suitors is to slaughter them all, in cold blood, while they are unarmed. Even those of us who think the death penalty is sometimes appropriate for misbehaving guests might find this problematic — while I think menacing servants, stealing food, and sexual harassment are serious matters, it's not clear to me that they merit being shot to death over 
dinner. Perhaps even more morally worrying, by contemporary standards, is Odysseus's treatment of the serving women who have been co-operating with the suitors: after the suitors are slaughtered, Odysseus and his son hang a large number of their female servants, on the spot and without anything resembling a trial, to punish them for collaboration.

However, even though this sort of mass slaughter might seem unjust or even cruel to many contemporary readers, it is easy to read Odysseus's avenging actions as not just appropriate but heroic in the world of The Odyssey. Odysseus's actions are painted in a positive light by Homer, and the scene is represented as a case of righteous vengeance and a heroic homecoming. Even the slaughter of the serving women is presented as disloyal subjects getting their comeuppance. We recognise that Homer approves; but more than that, we recognise that the world of Homer's epic is one where this sort of heroic action is appropriate and called for. I would guess even the Homeric gods approve of Odysseus's actions when he returns, and it is not because (perhaps against Homer's will) the gods themselves are making a mistake about what is called for. Of course we can read Homer against the grain, and interpret the scene as one of immoral and barbaric slaughter: but a very natural reading of these scenes is that in the story Odysseus is doing the right thing, because the values that govern the world of Homeric epic are not necessarily the values of the real world.

This is not an isolated case of behaviour that we would take to be immoral being portrayed as moral, heroic and praiseworthy in Homer's epics. Slave-taking is ubiquitous, and a fitting activity for heroes. While Agamemnon and Achilles may argue about the division of slaves, and there are clearly supposed to be right and wrong ways to divide up captured women, it is not questioned in the stories whether the whole institution of enslaving captured women is immoral, though it is presented as unfortunate for the slaves and a tragedy for their families. Even Hector, the paradigm hero, may well have taken slaves earlier in his career. I am not for a second suggesting that slavery is morally okay, but I do think it is natural to read Homeric epic so that slavery is (often) okay in the world of Homeric epic. And by this I do not just mean that the characters 
believe it is acceptable: it is rather that it is a different moral universe. ${ }^{5}$ One piece of evidence of this is that the gods themselves seem to have no problem with the institution per se, and another piece of evidence is the narrator taking the institution for granted. These do not settle the question about the norms of the epic, but in the absence of internal evidence pushing against this interpretation, I want to suggest this reading is a very natural one. If this reading is one that many of our contemporaries recognise and find natural, that suggests that they have not faced serious resistance to taking these moral claims to be true according to the Odyssey, or in the world of Homer's stories.

When discussing this case with philosophers, some have suggested that what Odysseus did was morally appropriate even by our standards. If so, the fact that we can easily read the story in a way that it is true that in the world of the story, Odysseus behaves correctly, would not provide an example where we read an alien moral code into a story without imaginative resistance. Let me say a little more in defence of my claim that that actions described are not morally correct according to our contemporary standards. (This conspiratorial "we" is meant to encompass most contemporary Western readers.) Odysseus and his family were in danger from the suitors, and we might well read the situation as being one where the suitors might overwhelm Odysseus if they were given the chance to arm themselves and prepare for a fight. So perhaps it could be argued that Odysseus has to massacre these suitors, as the only way of bringing them under control. But in the story, it is not clear that some halfway measure could not have worked: the final handful of suitors may have been willing to surrender, for example. And even if their misdeeds meant each and every suitor deserved execution, we might have some qualms about "due process", or a simpler bronze-age equivalent: perhaps the suitors that could have been captured ought to have been given an opportunity to say something in their own defence.

Even if we grant that his treatment of the suitors was somehow appropriate given his limited options, it is much harder to justify his slaughter out of hand of the Ithacan serving women. They were not a physical threat to him after the suitors were dealt with, and options such as exile were

\footnotetext{
5 Brock 2012 p 446 mentions another case from the Odyssey, in which Odysseus sacks a relatively inoffensive city, enslaves its women, and hands out the spoils "fairly". It is natural to read the story as being on in which that sort of aggression for gain is becoming for a king and hero.
} 
clearly more available once he resumed physical control of his palace. And even if some of them collaborated willingly, it is clear from the context that they were in the power of the suitors at least as much as Penelope was. The serving women were victims of threats of violence, or even worse, at the hands of the suitors, and did not have super-human powers to rise up and destroy their tormentors. And note again they are killed without having a chance to make a case for themselves, and without Odysseus calming down from his wrath to consider the situation with a level head. By our standards Odysseus's treatment of these relatively powerless servants is a horrifying act of domestic violence, not a heroic meting out of justice. I do not think we need to abandon our own values to read Homer's tale in the spirit in which it was told, and to think that in the world of the story Odysseus does something evil and contemptible is to read the story against the grain: but we should not get carried away here and think that Odysseus did something that was admirable by our lights.

The cases of The Odyssey and The Iliad are illustrative of many other cases in which we can get into the mindset of story-tellers from other times and other cultures, and should remind us that many of us are familiar with incorporating alien moral codes into what is true according to fictions from other times and other places. Medieval chivalric romances, stories glorifying colonialism and warfare from the nineteenth century, or even four-color comic book morality, all draw us into worlds where moral standards are different, and we can understand and even enjoy these stories while not taking the standards that are part of those stories to be part of the real world. Even though I am not a pacifist, I can appreciate a story world where it is always wrong for a hero to kill someone, and even though I have no brief for aristocracy, I can appreciate a story where a morally perfect knight keeps his serfs in poverty behind the scenes. In all of these cases we can adopt readings where we hold the actions of characters to our moral standards: but we can, and often do, adopt readings where alternative value systems are part of the world of the story, and we can do this without experiencing imaginative resistance.

Cases such as The Odyssey or the Aztec myth case are ones where the morals of peoples long ago are different. But I predict we will be able to find analogous cases for imaginative resistance due to other kinds of contents of stories, such as other aesthetic and evaluative differences. Stories in which scarification and tooth-filing make people beautiful are easier to swallow when 
they come from cultures that employ those beauty standards. Stories that treat something as funny, which to our sensibilities seems cruel or senseless can be approached better when we understand the sensibilities of the cultures they come from. (Some ancient Roman comedy is like this - it is hard to find it funny, but relatively easy to understand that it is funny in the story, once we have some intellectual understanding of their rather alien standards.) I do not know of cases analogous to Yablo's maple leaf case (a case from Yablo 2002 p 485 where a five-pointed jagged shape is nevertheless oval according to the story) from cultures with different geometrical understandings than ours, but there is perhaps a similar phenomenon when science-fiction writers play with non-Euclidean geometries, describing shapes that we would ordinarily think were impossible.

Notice that we do not need to wait until we find actual stories from other cultures to take advantage of this way of breaking down imaginative resistance with respect to various propositions. Creating stories and attributing them to distant or alien cultures will do as well. This can be done in the style of Liao et al 2004, who tell experimental participants that something is an Aztec creation myth when it is not (their vignette was made up), or it can be done by embedding a fiction within a fiction: telling an explicitly fictional story about a far away land or people, and say that they tell a certain story. This device might be useful for exploring fictions that have unusual parts: not just different moral or evaluative propositions, but propositions about shapes or linguistic meaning or other things which we might take for granted but about which the aliens or future philosophers or whoever hold a different view. When, according to the story, they tell stories about shapes or meanings or alternative mathematics or alternative logic, I predict suitably flexible readers will be able to understand those storieswithin-stories as reflecting the assumptions of their supposed authors.

A risk is that we might lose some of an audience's willingness to suspend disbelief if a story is only fictionally from another set of cultural assumptions: if Homer wants to tell the story of the serving women that's one thing, but if someone in our culture wants to tell a story of women getting righteously slaughtered for disobeying the head of the household, we might be suspicious about going along with it, even if they tell us it is a story told by people from far away. But my 
guess is that we can get a lot of this effect from framing stories as coming from other backgrounds once there's some intelligible reason why an author wants to do this.

\section{Using Genre to Break Down Imaginative Resistance}

Breaking down imaginative resistance by this sort of "cultural distancing" is only one of the techniques by which we can bring readers to find unusual propositions true according to fictions. (Liao et. al. themselves take it to be just one case of the influence of context and genre: Liao et. al. 2004 p 343-346.) Another important way we can vary what sorts of things are true according to a fiction, or true according to a reading of a fiction, is to vary genre expectations, and to introduce readers to new kinds of genres with new kinds of default assumptions about how to understand what is explicitly presented. Sometimes being presented with something startling or contrary-to-fact can be confusing for readers, but it is likely to be much less so if genre expectations are set appropriately. Indeed, some works belong to genres which encourage stretching what can be true according to stories, often beyond what is possible. Luis Borges's short stories are perhaps a paradigm example: in The Circular Ruins, for example, a wizard turns out to be a figment of his own imagination. Even Lewis Carroll's Alice has adventures beyond the possible: when the famous Cheshire cat disappears leaving nothing but his smile, he does not do this by leaving his teeth and lips: he does it the impossible way where all his body parts disappear but his smile remains. But in a surreal dreamscape, this should not surprise us nor be too difficult to understand.

Many artists of the last hundred years or so have been interested in pushing the boundaries of representation: and it should not be surprising that audiences interested in this enterprise should be willing to follow writers in taking all sorts of bizarre and impossible things to be true according to these stories. (The whole genre of "metafiction" plays with conventions to represent things that are often impossible or absurd.) This does not just go for written fictions either: experimental film, painting and many other arts could be deployed to represent things that most fictions rule out. Familiarise an audience with genre expectations that tell them to leave their usual assumptions about what the world of a fiction will be like at the threshold, and those audiences will be much more willing and able to recognise propositions true according to those 
fictions that they might normally resist. While I am not aware of genre signals to inform the reader that unusual moralities or aesthetic standards will govern the world of a fiction, the success of other genres in accustoming readers to impossibilities strongly suggests that we could establish conventions like that for a class of literature if we should wish.

Liao et al 2004 emphasise the influence of some genre shifts on imaginative resistance especially about moral matters. But this is far from the only way that we can exploit genre expectations to lower imaginative resistance. ${ }^{6}$ Some genres are especially welcoming to upending ordinary expectations of fiction or narration. These include magical realism, and fantastic tales such as the short stories by Borges. Many contemporary "metafictional" works, where characters do things like step out of their stories, or upbraid their authors, undermine our expectations of how the usual limits of stories can apply. Surreal art and literature also pushes against the boundaries of what we would ordinarily take for granted in interpreting artworks, including fictions of all sorts. And some precursors of these transgressive fictions are widely consumed: as mentioned above, Lewis Carroll's Alice in Wonderland stories frequently involve impossible goings-on, including things that seem like straightforward conceptual impossibilities, such as the Cheshire Cat's gradual disappearance leaving only his smile. (I take it is impossible for a smile to be present without a mouth or a face some other underlying substrate.)

I do not know of any generally accepted name for this collection of genres that are particularly suited to overturning or suspending ordinary principles that we might normally take to be true in fictions we encounter. "Conceptual fiction" might have been a good label if that were not already in use for another category in literary theory. I plan to use the expression "philosophical fiction" for the general category, though I want to disavow any suggestion that those who produce these fictions have particularly philosophical aims in mind or do so as part of a philosophical project.

\footnotetext{
${ }^{6}$ Brock 2012 employs genre conventions in a different way to explain imaginative resistance in the case of "morally deviant" fictions. He suggests that it is normally a convention that an author will attempt to make the moral principles true in a fiction the same as the moral principles which in fact obtain. Because of this convention, readers will not even notice readings where the moral principles in a story might be only fictional. If Brock is right about actual cases of imaginative resistance when faced e.g. by morally deviant fictions, I think it should be a relatively straightforward matter to introduce new genre conventions covering "moral fiction" where readers no longer assume they are to match the fictional moral truths to the actual moral truths. (I suspect, however, that Brock's proposal would have to be modified to take into account the existing willingness of readers to take the morality of the story to be different from that of the real world, as already discussed above.)
} 
Those unfamiliar with these genres may well face imaginative resistance when first exposed to them: though I suspect many people find their way into these genres without much refusal to take the story at face value when it describes impossible things, as opposed to taking the tales to be interesting or wondrous. (To take another example from Borges, in The Aleph the protagonist discovers a strange object that contains the whole universe within it_including the object itself, as a proper part. I have never seen the reaction that Borges is somehow mis-describing what is going on in the world of the story, even from those who find it mind-bending. I can see how a philosopher committed to thinking that this is not a correct reading of the story could defend an alternative, but if someone claimed to be unable to read the story the way Borges apparently intends I would suspect an ulterior motive for that resistance.)

It may well be that philosophical fictions to date have not engaged in suspending or contradicting every principle that we might normally use to guide us in telling what is true in a fiction. And perhaps trying to suspend them all at once would be impractical, or at least not be conducive to producing interesting fictions. Still, it is tempting to think that if there are as-yet-unplayed with principles, then it is only a matter of time before a fiction writer will attempt something interesting by flouting those principles, and the track record of writers such as Borges suggests that skilled attempts to do so may well succeed. (Some of doing so may be as simple as the author's drawing the relevant principle to the readers' attention and saying explicitly that this is not what is going on.) In this way, the limits of fiction may be similar to the limits of art: the history of art in the twentieth century should teach us that when an orthodoxy holds that there are certain limits to what art can do, experimental artists attempt to transcend those limits, and often succeed.

I should be clear that I am not arguing that there are no limits to what we can make true according to fictions. Human brains are finite, and the time we have to tell stories is finite, so it would be surprising if we could make any proposition distinctively fictional no matter how complicated, or alien. A story of the form "Then the great figure started to pronounce digits: 1, 0 , $1,1,0,1,0,0,1,1 \ldots$ " could be extended in infinitely many ways just with ones and zeroes, and there are particular sequences of ones and zeros we have no very good way to uniquely specify with finite resources. I will therefore restrict my claim to propositions that can be understood by 
readers. My claim is that for any such propositions that are understandable, (inside or outside fiction) readers can be brought to take such a proposition to be true according to an available reading of a fiction, without imaginative resistance. For many interesting propositions, this may require sophistication on the part of readers, including familiarity with "philosophical fiction" genres, and/or an ability to read their way into fictions written (or supposedly written) by authors who make very different assumptions about the world from the ones readers are used to.

Our limits in specifying particular propositions may not limit our ability to make propositions true according to stories if we help ourselves to more powerful techniques such as quantification. "Dr Logic's plan to conquer the world took an unexpected turn when the carefully contained contradiction escaped. Once it reached the classical logic engines, everything whatsoever became the case, rendering Dr Logic's world trivial." By the end of the story, was it the case that every proposition is true according to the story of Dr Logic? If so, then perhaps this sort of technique is a powerful one for demonstrating fictions where various recalcitrant propositions are taken to be true without resistance.

To the extent that imaginability requires more than a proposition being true according to a story that we can understand (or a reading of that story), there may well be tighter constraints on imaginability. I hesitate to wade very far into the waters here, since I suspect disputes about "imaginability" are to some extent verbal: it seems to me that different theorists have identified different phenomena of interest, with more or less clarity, and labelled those phenomena "imaginability". Instead, let me make the relatively banal point that insofar as it is sufficient for something to be imaginable that one can take it to be true according to a story, if the account I offer is right then any proposition which is intelligible is imaginable.

\section{Empirical Testing?}

In this paper I am defending a psychological hypothesis: that for any understandable proposition $p$, we will be able to find or construct a fiction so that a suitably cosmopolitan reader can be brought to take it to be true according to a fiction (i.e. some fiction or other) without imaginative resistance. So it might be reasonable to ask how we might scientifically test whether this generalisation is true. Experiments like those reported by Liao et al 2004 are a good start: and I 
conjecture results similar to theirs can be established for non-moral propositions that typically face imaginative resistance, through a technique similar to theirs.

It will be harder to directly test the central claim made by this paper in laboratory conditions. The claim is that a course of cultural expansion of horizons, familiarisation with philosophical views and other ideologies far from one's own, and an exposure to a wide range of contemporary genres of fiction will put people in a position to take any intelligible proposition $p$ as true in some story or other (or at least they will be in a position to do that relative to some reading or other of that story they engage in). The kind of familiarisation envisaged is hard to create in an experimental subject in an hour or two. It is, however, the kind of experience that is produced by some, but not all, undergraduate and graduate programs in the humanities, and we do have groups available for study with the right kind of preparation. While degrees in literature or anthropology or philosophy are not guarantees that someone has had the required breadth of exposure, we could, if we wished, test groups with training and expertise in these areas. These groups could be tested for whether they are able to see fictions with the right background filled in as having as part of them propositions that might normally go against the grain. In advance of experimental testing of such subjects, we might take note of the range of speculative fiction and understanding for a wide variety of views alien from our own, and ask ourselves whether we can understand stories that accidentally or by design overturn comfortable default assumptions we might otherwise make about what is going in in fictions. While more systematic investigation of responses to such fiction is worthwhile, I think it is already plausible that widened intellectual horizons, whether through understanding of other cultural assumptions, or others' outré viewpoints, or familiarity with the flexibility of fiction and its genres, can lead to being able to understand more things being parts of stories than one would otherwise. In my experience this process has very few limits, and fallible evidence for that is how far people can already go.

If we were to test this, either through tests of suitably educated humanists or corpus-searches of experimental fiction and stories from culturally alien authors, we should keep in mind that different experiences with fiction may yield different propensities to avoid imaginative resistance. We should also keep in mind that readers have some agency in which readings they choose to indulge in, and we should not be over-hasty in inferring, from that fact that they 
choose a reading that resists taking $p$ to be true according to a story, that they cannot or will not also recognise another reading that does include $p$. But of course it is a commonplace of social science research that experimenters must be sensitive to a range of potential confounds when designing tests or data searches. So while testing is not entirely straightforward, it is not impossible either. In the meantime, I can report that I can get myself in the frame of mind where various supposedly difficult propositions are naturally true according to available readings of suitably constructed fictions. This testimony might be suspect, since sceptics might reasonably think my theoretical commitments could lead me astray, or render me sufficiently unlike ordinary readers to be the basis for generalisation. Still, I think many readers of this paper will be able to get themselves in a similar frame of mind, after reflecting on the range of philosophical fiction and the flexibility of story-telling: so in advance of social science research, you, dear reader, may be able to detect the effect yourself.

\section{Lessons}

Almost any understandable proposition can be embedded in a fiction in a way that it will not evoke imaginative resistance in typical consumers of that fiction. This is not to deny that imaginative resistance occurs: it can occur for mundane reasons, or for more philosophically interesting reasons, including the embedding of impossibilities, immoral standards, or even baffling incongruities in a story.

The fact that any understandable proposition can be embedded in a way that it does not evoke significant imaginative resistance, with the right cues from genre and sufficient literary cosmopolitanism from an audience, strongly suggests that we will not be able to learn much about the limits of possibility, or the deeply held normative commitments of readers, or substantive limits on conceivability, by testing which fictions evoke imaginative resistance. ${ }^{7}$

\footnotetext{
${ }^{7}$ Two other options are worth mentioning: perhaps fictionality (or imaginability or conceivability) lines up with possibility, but it is just that possibility is much more generous than we might have thought. One way this might be is if every proposition is possible: see Mortensen 1989 for a defence of this view. Or perhaps there is a very generous kind of possibility, literary possibility, so that everything that can be true in a story is possible in this sense of possibility (see Nolan 2015 section 5). I do not yet see enough motivation to be revisionary about possibility in either of these ways: but perhaps some of those deeply invested in a link between conceivability and possibility may wish to react to the discovery that far more than we might have thought is conceivable with the response that, after all, far more than we might have thought is indeed possible.
} 
Explanations of non-mundane imaginative resistance in terms of unfamiliarity with certain kinds of content, or a lack of experience with certain kinds of genres, undercut the main extant theories that suggest imaginative resistance could track the bounds of what is possible, or even the bounds of what readers think is possible. The first, negative, conclusion of this paper is that our capacity to engage non-resistantly with a given scenario or given proposition in fiction is little or no help in determining whether that scenario or proposition is a possible one.

If I am right about any entertainable proposition being able to be taken to be part of a story without imaginative resistance, that suggests two positive conclusions about wider philosophical matters. As mentioned above, some theorists (Stalnaker 1984, Lewis 1986, Jackson 2011) have wanted to tie the limits of representation to the limits of possibility. In a straightforward possible worlds theory of propositions, for example, each proposition is associated with a distinct set of possible worlds, and there is only one impossible proposition (the set of no worlds). That impossible proposition entails every other proposition, and despite appearances there is only one proposition that cannot be true. According to that theory, there is no scope for taking one impossibility to be true according to a fiction without taking all impossibilities to be part of the story, and arguably no way of taking one to be part of a story without taking the story to be entirely trivial, having all propositions true according to it. All of the authors mentioned have suggested various manoeuvers to save the phenomena: perhaps we discriminate different sentences that express the same impossible propositions (Stalnaker 1984 71-87), or perhaps we employ a "two dimensional" semantics, considering a range of possible-worlds propositions that could be expressed by a sentence much like our actual sentences (Jackson 2011). None of these approaches seem adequate to me to capture the phenomena, though there is not room to argue for this convincingly here. (Lewis 2004 seems to concede something additional is needed, though alas his untimely death prevented him from exploring what the best way is to develop his views in the light of puzzles about impossible fictions.)

The second positive suggestion is that rather more propositions are conceivable than some theorists would wish to concede. Propositions that can be taken to be true according to a fiction can usually be caught up in an interesting network of attitudes: we can use the fact that $p$ is part of a fiction to anticipate what else might be part of it; we can use that information to engage in 
aesthetic evaluations of the fiction; we can think about variations where alternatives to $p$ were chosen, and so on. Such propositions seem to pass a lot of tests for being representable in some rich sense and being able to play significant cognitive roles. Several philosophers have wanted to use conceivability as their key guide to metaphysical possibility and metaphysical necessity (see e.g. Yablo 2002 and Chalmers 2002, among many others). Writers like Yablo and Chalmers have wanted conceivability to serve as a diagnosis not just of possibility but of impossibility: at a first pass, being able to conceive $p$ is strong evidence for the metaphysical possibility of $p$, and being unable to conceive of $q$ is strong evidence for the impossibility of $q$.

Much of the literature on using conceivability as a guide to possibility involves explaining away apparent counterexamples: tweaking what the exact connection is so that various things that seem conceivable are not conceivable in the right way (or are not "ideally conceivable"), or so that various propositions that seem straightforwardly possible though inconceivable turn out to not be counterexamples to the refined principles. So even if it is conceded that we can take a wide range of impossibilities to be true according to fictions, those seeking a close link between conceivability and possibility will still have plenty of room to move. ${ }^{8}$ The plausibility of there being a tight link between conceivability and possibility still comes under pressure when what is prima facie conceivable comes apart a long way from what is prima facie possible, however, even if a theory with enough qualifications and hedges can avoid being counter-exampled outright.

The fact that so many kinds of propositions can be treated as true according to fictions by readers with suitably wide horizons is thus grist to the mill for the claim that a wide range of impossibilities are conceivable, in the main sense of "conceivable" of interest to philosophy and modal epistemology. While I think there are salvageable links between some cases of conceiving and some sorts of reasons to take propositions to be possible, nothing so ambitious as the claim that almost everything that is conceivable is possible is likely to survive. The fact that such a

\footnotetext{
${ }^{8}$ Levin 2012, for example, offers an attempt to hold on to a conceivability-possibility link even in the face of apparently being able to take impossible things to be true according to stories, for example. She claims the scenarios we are unable to imagine are "just those whose depiction seems to involve conceptual incoherence" (p 394). Assuming a tight imaginability-truth in fiction link, as she also seems to do, this seems to me to veer very close to just denying the data: as indicated above, there are just too many cases in philosophical fiction where competent readers take things to be true in a story which are not even conceptual possibilities.
} 
wide range of impossibilities are conceivable instead supports views such as that defended by (Priest 2016) that every understandable proposition is conceivable. If this is right, the role of conceivability in modal epistemology is limited, at best.

Sometimes some readers do refuse to accept readings of fictions, and not just for mundane reasons like a lack of interest or a lack of attention. Imaginative resistance is a real phenomenon, and further investigation of it is likely to yield interesting results about the psychology and norms of uptake of fiction. But some central philosophical approaches to it thus far have been importantly misguided in one respect: there is not some special class of propositions, or small sets of propositions, that are intelligible but are also once-and-for-all propositions that will evoke imaginative resistance in any fiction in which they occur. Readers with suitably broad horizons, who are familiar with assumptions that other cultures made or might make, and familiar with a range of genres including the transgressive "philosophical fictions" mentioned above, will be able to see relatively straightforward readings of fictions where such propositions are parts of the story. This is good news for a nuanced understanding of imaginative resistance, but bad news for some of the conclusions philosophers have drawn from the phenomenon of imaginative resistance. It will not provide us much of a straightforward guide to what is metaphysically possible, or what our normative commitments are, and if it is anything like a straightforward guide to what is conceivable, it shows that what we can conceive far outruns what is plausible metaphysically possible or even conceptually coherent. Our power to create and understand fictions far outstrips what is dreamt of in more conservative philosophies of fiction. ${ }^{9}$

Daniel Nolan University of Notre Dame University of Queensland E-mail: dnolan2@nd.edu

\footnotetext{
9 Thanks to Tamar Gendler, Rod Girle and audiences at the Conceivability and Modality conference at the Sapienza University of Rome, and the University of Queensland, for discussion and feedback on ancestors of this paper. Thanks also to anonymous referees of ancestors of this paper, and to comments from Joseph Salerno and an anonymous referee on the current version. Some of the research for this paper was carried out while I was based at the University of Queensland.
} 


\section{References}

Stuart Brock. 2012. "The Puzzle of Imaginative Failure." Philosophical Quarterly 62.248: 443463.

David Chalmers. 2002. "Does Conceivability Entail Possibility?" In Conceivability and Possibility, edited by Tamar Gendler and John Hawthorne. Oxford: Oxford University Press, 145-200.

Judith Fetterley. 1978. The Resisting Reader: A Feminist Approach to American Fiction. Indianapolis: Indiana University Press.

Tamar Gendler. 2000. "The Puzzle of Imaginative Resistance." Journal of Philosophy 97.2: 5581.

Tamar Gendler. 2006. "Imaginative Resistance Revisited" in The Architecture of the Imagination edited by Shaun Nichols. Oxford: Oxford University Press, pp 149-173.

Tamar Gendler and Shen-yi Liao. 2016. "The Problem of Imaginative Resistance." In The Routledge Companion to the Philosophy of Literature, edited by Noël Carroll and John Gibson. London: Routledge, 405-418.

F.C. Jackson. 2011. "Possibilities for Representation and Credence: Two Space-ism versus One Space-ism.", in Epistemic Modality, edited by Andy Egan and Brian Weatherson. Oxford: Oxford University Press, 131-143.

Janet Levin. 2012. "Imaginability, Possibility, and the Puzzle of Imaginative Resistance." Canadian Journal of Philosophy 41.3: 391-422.

David Lewis. 1986. On The Plurality of Worlds. Oxford: Blackwell. 
David Lewis. 2004. "Letters to Beall and Priest." in Graham Priest, Jc Beall, and Bradly Armor Garb, (eds) The Law of Non-Contradiction. Oxford: Oxford University Press. 176-177.

Shen-yi Liao, Nina Strohminger, and Chandra Sripada. 2014. "Empirically Investigating Imaginative Resistance." British Journal of Aesthetics 54.3: 339-355.

Christopher Mortensen. 1989. "Anything is Possible." Erkenntnis 30: 319-337.

Daniel Nolan. 2015. "Personification and Impossible Fictions." British Journal of Aesthetics 55.1: 57-69.

Graham Priest. 1997. "Sylvan's Box: A Short Story and Ten Morals." Notre Dame Journal of Formal Logic 38.4: 573-582.

Graham Priest. 2016. "Thinking the Impossible." Philosophical Studies 173: 2649-2662.

Robert Stalnaker. 1984. Inquiry. Cambridge: MIT Press.

Nils-Hennes Stear. 2015. "Imaginative and Fictionality Failure: A Normative Approach." Philosophers Imprint 15.34: 1-18.

C.S. Todd. 2009. "Imaginability, Morality and Fictional Truth: Dissolving the Puzzle of 'Imaginative Resistance'." Philosophical Studies 143.2: 187-211.

Peter van Inwagen. 1993. "Naive Mereology, Admissible Valuations, and Other Matters." Nô̂s 27.2: 229-234.

Kendall Walton. 1994. "Morals in fiction and fictional morality." Supplement to the Proceedings of the Aristotelian Society 68: 27-50.

Brian Weatherson. 2004. "Morality, Fiction, and Possibility." Philosophers' Imprint 4.3: 1-27. 
Stephen Yablo. 2002. "Coulda, Woulda, Shoulda." in Conceivability and Possibility edited by Tamar Gendler and John Hawthorne. Oxford: Oxford University Press. 441-492. 